\title{
A SIMPLE PROOF OF DUALITY THEOREM FOR MONGE-KANTOROVICH PROBLEM
}

\author{
Toshio Mikami
}

To the memory of the late Professor Nobuyuki Suita

\begin{abstract}
We give a simple proof of the duality theorem for the Monge-Kantorovich problem in the Euclidean setting. The selection lemma which is useful in the theory of stochastic optimal controls plays a crucial role.
\end{abstract}

\section{Introduction}

Let $P_{0}$ and $P_{1}$ be Borel probability measures on $\mathbf{R}^{d}$ and $\mathscr{A}\left(P_{0}, P_{1}\right)$ denote the set of all $\mu \in \mathscr{M}_{1}\left(\mathbf{R}^{d} \times \mathbf{R}^{d}\right)$ for which $\mu\left(d x \times \mathbf{R}^{d}\right)=P_{0}(d x)$ and $\mu\left(\mathbf{R}^{d} \times d x\right)=$ $P_{1}(d x)$, where $\mathscr{M}_{1}\left(\mathbf{R}^{d} \times \mathbf{R}^{d}\right)$ denotes the complete separable metric space, with a weak topology, of Borel probability measures on $\mathbf{R}^{d} \times \mathbf{R}^{d}$ (see e.g. [1]). Take also a Borel measurable $c(\cdot, \cdot): \mathbf{R}^{d} \times \mathbf{R}^{d} \mapsto[0, \infty)$.

The study of a minimizer of the following $\mathscr{T}\left(P_{0}, P_{1}\right)$ is called the MongeKantorovich problem which has been studied by many authors and which has been applied to many fields (see [2, 4, 6, 9, 10] and the references therein):

$$
\mathscr{T}\left(P_{0}, P_{1}\right):=\inf \left\{\int_{\mathbf{R}^{d} \times \mathbf{R}^{d}} c(x, y) \mu(d x d y) \mid \mu \in \mathscr{A}\left(P_{0}, P_{1}\right)\right\} .
$$

The duality theorem for $\mathscr{T}\left(P_{0}, P_{1}\right)$ plays a crucial role in the proof of the Monge-Kantorovich problem and has been proved for a wide class of functions $c(\cdot, \cdot)$ (see $[5,8-10])$.

They say that the duality theorem for $\mathscr{T}\left(P_{0}, P_{1}\right)$ holds if

$$
\mathscr{T}\left(P_{0}, P_{1}\right)=\sup \left\{\int_{\mathbf{R}^{d}} \psi(1, y) P_{1}(d y)-\int_{\mathbf{R}^{d}} \psi(0, x) P_{0}(d x)\right\},
$$

where the supremum is taken over all $\psi(t, \cdot) \in L^{1}\left(P_{t}\right)(t=0,1)$ for which

Partially supported by the Grant-in-Aid for Scientific Research, No. 15340047, 15340051 and 16654031, JSPS.

Received December 14, 2004; revised June 10, 2005. 


$$
\psi(1, y)-\psi(0, x) \leq c(x, y) .
$$

In [7] we obtained a stochastic control version of (1.2)-(1.3) and gave a new approach to $h$-path processes for diffusion processes as its application. We also showed that its zero noise limit yields (1.2)-(1.3) (see [8]).

In this paper we give a simple proof for (1.2)-(1.3) since known proofs for (1.2)-(1.3) are complicated. Indeed, Kellerer used a functional version of Choquet's capacitability theorem (see [5] and also [9]) and Villani did a minimax principle (see [10, sect. 1.1]).

Our proof relies on the Legendre duality of a lower semicontinuous convex function of Borel probability measures on $\mathbf{R}^{d}$ and on the selection lemma which is useful in the theory of stochastic optimal controls (see the proof of Theorem 2.1).

I would like to dedicate this paper to the late Professor Nobuyuki Suita who used to be very nice to me.

\section{A simple proof for Duality Theorem}

We first describe our assumption.

(A.1) $c \in C\left(\mathbf{R}^{d} \times \mathbf{R}^{d}:[0, \infty)\right)$ and $c(x, y) \rightarrow \infty \quad$ as $|y-x| \rightarrow \infty$, and $\inf \left\{c(x, y) \mid y \in \mathbf{R}^{d}\right\}$ is bounded.

(A.2) $\mathscr{T}\left(P_{0}, P_{1}\right)$ is finite.

(A.3) $P_{0}$ is absolutely continuous with respect to the Lebesgue measure $d x$.

We give a simple proof to the following which can be obtained from the known result (see [5] and also [9, 10]).

TheOREM 2.1 (Duality Theorem). Suppose that (A.1)-(A.3) hold. Then (1.2)-(1.3) holds.

Proof. We prove (1.2), where the supremum is taken over all $\psi(t, \cdot) \in$ $C_{b}\left(\mathbf{R}^{d}\right)(t=0,1)$ for which $(1.3)$ holds. This implies the duality theorem for $\mathscr{T}\left(P_{0}, P_{1}\right)$ since $(1.2)-(1.3)$ with " $=$ " replaced by " $\geq$ " holds and since $C_{b}\left(\mathbf{R}^{d}\right) \subset$ $L^{1}\left(P_{t}\right)(t=0,1)$.

The proof is divided into the following (2.1)-(2.3):

$$
\mathscr{T}\left(P_{0}, P_{1}\right)=\sup _{f \in C_{b}\left(\mathbf{R}^{d}\right)}\left\{\int_{\mathbf{R}^{d}} f(y) P_{1}(d y)-\mathscr{T}_{P_{0}}^{*}(f)\right\},
$$

where for $f \in C_{b}\left(\mathbf{R}^{d}\right)$,

$$
\mathscr{T}_{P_{0}}^{*}(f):=\sup _{P \in \mathscr{M}_{1}\left(\mathbf{R}^{d}\right)}\left\{\int_{\mathbf{R}^{d}} f(y) P(d y)-\mathscr{T}\left(P_{0}, P\right)\right\} .
$$

For $f \in C_{b}\left(\mathbf{R}^{d}\right)$,

$$
\varphi(x ; f):=\sup _{y \in \mathbf{R}^{d}}\{f(y)-c(x, y)\} \in C_{b}\left(\mathbf{R}^{d}\right),
$$




$$
\mathscr{T}_{P_{0}}^{*}(f)=\int_{\mathbf{R}^{d}} \varphi(x ; f) P_{0}(d x) .
$$

We first prove (2.1). We only have to prove $\mathscr{T}\left(P_{0}, \cdot\right): \mathscr{M}_{1}\left(\mathbf{R}^{d}\right) \mapsto[0, \infty]$ is lower semicontinuous and convex. Indeed, this and (A.2) implies (2.1) from [1, Theorem 2.2.15 and Lemma 3.2.3], by putting $\mathscr{T}\left(P_{0}, P\right)=\infty$ for $P \notin \mathscr{M}_{1}\left(\mathbf{R}^{d}\right)$.

Suppose that $Q_{n} \rightarrow Q$ weakly as $n \rightarrow \infty$. Then it is easy to see that $\bigcup_{n \geq 1} \mathscr{A}\left(P_{0}, Q_{n}\right)$ is tight in $\mathscr{M}_{1}\left(\mathbf{R}^{d}\right)$. Take $\mu_{n} \in \mathscr{A}\left(P_{0}, Q_{n}\right)(n \geq 1)$ for which

$$
\mathscr{T}\left(P_{0}, Q_{n}\right)+\frac{1}{n}>\int_{\mathbf{R}^{d} \times \mathbf{R}^{d}} c(x, y) \mu_{n}(d x d y) \geq \mathscr{T}\left(P_{0}, Q_{n}\right) .
$$

For any convergent subsequence $\left\{\mu_{k(n)}\right\}_{n \geq 1}$ of $\left\{\mu_{n}\right\}_{n \geq 1}$ and its weak limit $\mu_{0}$, $\mu_{0} \in \mathscr{A}\left(P_{0}, Q\right)$ and

$$
\liminf _{n \rightarrow \infty} \int_{\mathbf{R}^{d} \times \mathbf{R}^{d}} c(x, y) \mu_{k(n)}(d x d y) \geq \int_{\mathbf{R}^{d} \times \mathbf{R}^{d}} c(x, y) \mu_{0}(d x d y)
$$

since $c \geq 0$ from (A.1). Hence $\mathscr{T}\left(P_{0}, \cdot\right): \mathscr{M}_{1}\left(\mathbf{R}^{d}\right) \mapsto[0, \infty]$ is lower semicontinuous. $\mathscr{T}\left(P_{0}, \cdot\right): \mathscr{M}_{1}\left(\mathbf{R}^{d}\right) \mapsto[0, \infty]$ is also convex since for any $P, Q \in \mathscr{M}_{1}\left(\mathbf{R}^{d}\right)$ and $\lambda \in(0,1)$,

$$
\left\{\lambda \mu+(1-\lambda) v \mid \mu \in \mathscr{A}\left(P_{0}, P\right), v \in \mathscr{A}\left(P_{0}, Q\right)\right\} \subset \mathscr{A}\left(P_{0}, \lambda P+(1-\lambda) Q\right) .
$$

We next prove (2.2). From (A.1), for $f \in C_{b}\left(\mathbf{R}^{d}\right)$ and $x \in \mathbf{R}^{d}$,

$$
-\infty<\inf _{y \in \mathbf{R}^{d}} f(y)-\sup _{x \in \mathbf{R}^{d}}\left\{\inf _{y \in \mathbf{R}^{d}} c(x, y)\right\} \leq \varphi(x ; f) \leq \sup _{y \in \mathbf{R}^{d}} f(y)<\infty,
$$

which implies that $\varphi(\cdot ; f)$ is bounded.

From (A.1), the following set is not empty for any $x \in \mathbf{R}^{d}$ and is bounded on every bounded subset of $\mathbf{R}^{d}$ :

$$
D_{x}:=\left\{y \in \mathbf{R}^{d} \mid \varphi(x ; f)=f(y)-c(x, y)\right\} .
$$

Suppose that $x_{n} \rightarrow x$ as $n \rightarrow \infty$. Take $y_{n} \in D_{x_{n}}$ and $y \in D_{x}$. Then there exist a convergent subsequence $\left\{y_{k(n)}\right\}_{n \geq 1}$ and $\tilde{y}$ such that $y_{k(n)} \rightarrow \tilde{y}$ as $n \rightarrow \infty$ and such that

$$
\begin{aligned}
\limsup _{n \rightarrow \infty} \varphi\left(x_{n} ; f\right) & =\lim _{n \rightarrow \infty}\left\{f\left(y_{k(n)}\right)-c\left(x_{k(n)}, y_{k(n)}\right)\right\} \\
& =f(\tilde{y})-c(x, \tilde{y}) \leq \varphi(x ; f) .
\end{aligned}
$$

The following together with (2.8) implies that $\varphi(\cdot ; f) \in C\left(\mathbf{R}^{d}\right)$ :

$$
\liminf _{n \rightarrow \infty} \varphi\left(x_{n} ; f\right) \geq \lim _{n \rightarrow \infty}\left\{f(y)-c\left(x_{n}, y\right)\right\}=\varphi(x ; f) .
$$

We prove (2.3) to complete the proof. For $f \in C_{b}\left(\mathbf{R}^{d}\right)$, 


$$
\begin{aligned}
\mathscr{T}_{P_{0}}^{*}(f) & =\sup _{P \in \mathscr{M}_{1}\left(\mathbf{R}^{d}\right)}\left\{\sup \left\{\int_{\mathbf{R}^{d} \times \mathbf{R}^{d}}(f(y)-c(x, y)) \mu(d x d y) \mid \mu \in \mathscr{A}\left(P_{0}, P\right)\right\}\right\} \\
& \leq \int_{\mathbf{R}^{d}} \varphi(x ; f) P_{0}(d x) .
\end{aligned}
$$

(A.1) implies that the set $\bigcup_{|x| \leq r}\left(\{x\} \times D_{x}\right)$ is compact for any $r>0$. Indeed, the set $\bigcup_{|x| \leq r}\left(\{x\} \times D_{x}\right)$ is bounded as we mentioned in (2.7) and is closed since the set $D:=\left\{(x, y) \in \mathbf{R}^{d} \times \mathbf{R}^{d} \mid \varphi(x ; f)=f(y)-c(x, y)\right\}$ is closed from (2.2) and since

$$
\bigcup_{|x| \leq r}\left(\{x\} \times D_{x}\right)=D \cap\left\{(x, y) \in \mathbf{R}^{d} \times \mathbf{R}^{d}|| x \mid \leq r\right\} .
$$

Hence there exists a measurable function $u: \mathbf{R}^{d} \mapsto \mathbf{R}^{d}$ such that $u(x) \in D_{x}, d x$-a.e. by the selection lemma (see [3, p. 199]). In particular, from (A.3) and (2.10),

$$
\mathscr{T}_{P_{0}}^{*}(f) \leq \int_{\mathbf{R}^{d} \times \mathbf{R}^{d}}\{f(y)-c(x, y)\} P_{0}(d x) \delta_{u(x)}(d y) \leq \mathscr{T}_{P_{0}}^{*}(f) . \quad \text { Q.E.D. }
$$

\title{
REFERENCES
}

[1] J. D. Deuschel and D. W. Stroock, Large deviations, Pure and applied mathematics 137, Academic Press, Inc., Boston, MA, 1989.

[2] L. C. Evans, Partial differential equations and Monge-Kantorovich mass transfer, Current developments in mathematics, 1997, Int. Press and Cambridge, Boston, MA, 1999, 65-126.

[ 3 ] W. H. Fleming and R. W. Rishel, Deterministic and stochastic optimal control, SpringerVerlag, Berlin, Heidelberg, New York, Tokyo, 1975.

[4] W. Gangbo and R. J. McCann, The geometry of optimal transportation, Acta Math. 177 (1996), 113-161.

[ 5 ] H. G. Kellerer, Duality theorems for marginal problems, Z. Wahrsch. Verw. Gebiete 67 (1984), 399-432.

[6] T. Mikami, Monge's problem with a quadratic cost by the zero-noise limit of $h$-path processes, Probab. Theory Related Fields 129 (2004), 245-260.

[7] T. Mikami and M. Thieullen, Duality theorem for stochastic optimal control problem, Hokkaido University preprint series 652, 2004.

[8] T. Mikami and M. Thieullen, Optimal transportation problem by stochastic optimal control, Hokkaido University preprint series 690, 2005.

[9] S. T. RACHEV AND L. RÜSCHENDORF, Mass transportation problems, I: Theory, II: Application, Springer-Verlag, Berlin, Heidelberg, New York, Tokyo, 1998.

[10] C. Villani, Topics in optimal transportation, Graduate studies in mathematics 58, Amer. Math. Soc., Providence, RI, 2003.

\author{
Department of Mathematics \\ HOKKAIDO UNIVERSITY \\ SAPPORO 060-0810 \\ JAPAN \\ E-mail: mikami@math.sci.hokudai.ac.jp
}

